Perspective

\title{
Homeopathy workshops
}

\section{Introduction}

Homeopathy, now considered an alternative medical approach, is a system of medicine based on pharmacology and toxicology of natural substances. Experimentation and "Provings" of these substances have shown effects on physical, physiological, and psychological aspects of an individual. Homeopaths believe in treating the individual rather than combating the disease. Homeopathic medicines are the diluted forms of substances found in nature and are used for different types of acute and chronic illnesses in many different settings. Some of the illnesses treated by conventional medicine and even some illnesses that don't have a conventional medical treatment can be treated by Homeopathy. Safety of Homeopathic medicines makes them a suitable treatment choice during pregnancy, breastfeeding as well as for the growing child. There is growing body of scientific research supporting the effectiveness of Homeopathy.

\section{Origins of homeopathy}

Any innovation in history has started by pushing the limits and thinking outside the box. Samuel Hahnemann (1755-1843) a German Physician, linguist, and the father of Homeopathy noticed that the medical practices of his time were crude and produced more harm than cure. This is the time when ether and chloroform were used as anesthetics in surgical units with lots of side effects, and mercury was used to treat syphilis, with treatment being worse than the disease (Figure 1).

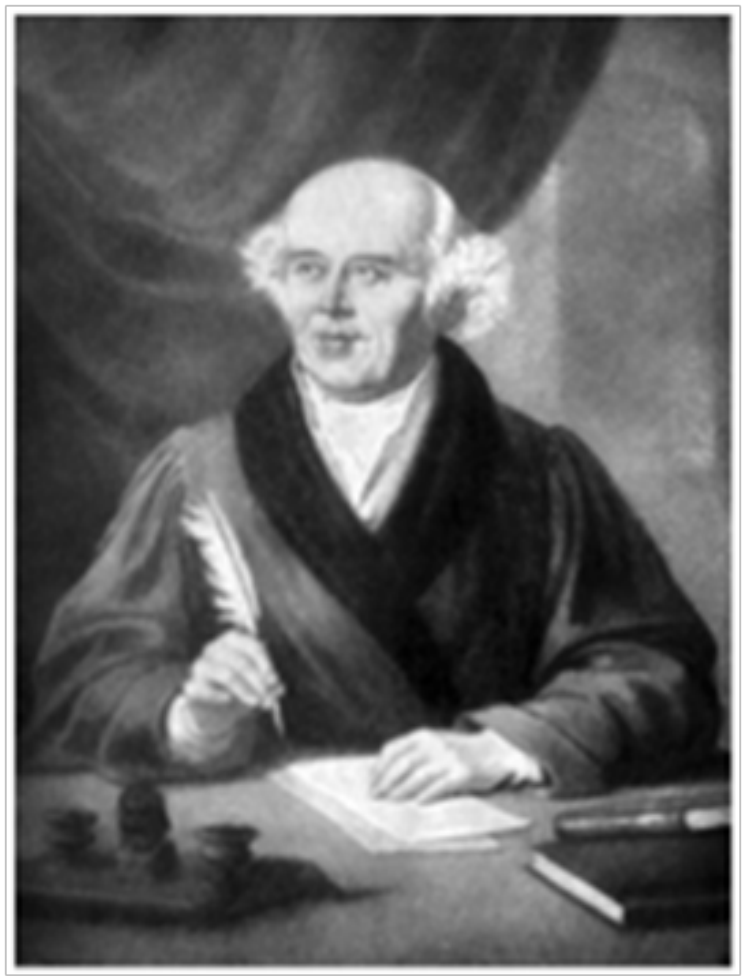

Figure I Samuel Hahnemann.
Volume I Issue 5 - 2015

\author{
Payam Hakimi \\ Center for Education and Development of Homeopathy, Body \\ of Harmony Institute of Health \& Healing, USA
}

\begin{abstract}
Correspondence: Payam Hakimi, Center for Education and Development of Homeopathy, Body of Harmony Institute of Health \& Healing, I44 South Beverly Drive, Suite 400 Beverly Hills, CA 90212, USA, Email doctor.hakimi@gmail.com
\end{abstract}

Received: July 10, 2015 | Published: September 16, 2015

Based on an observation made earlier by Paracelsus that "what makes a man ill also cures him", Hahnemann started experimenting with minimal amount of substances to cure illnesses. He came across this concept when translating a medical treatise on the effectiveness of Cinchona's (Quinine) bark in treating the symptoms of Malaria. His experimentation with ingesting this substance caused shivering, fever, and joint pains, similar to the symptoms of Malaria.

He then started experimenting with different substances available in nature on himself and other healthy individuals to establish their effects. He called these experiments Provings. Provings are collected in large Homeopathic reference guide called the Materia Medica. Provings are still compiled by dosing healthy individuals with various substances and documenting the results.

Some of the medications that are used in conventional medicine today are used based on their provings from the Homeopathic Materia Medica, like Digitalis (foxglove plant), a medication used for heart rhythm abnormalities, and Nitroglycerin for blood pressure issues (Figure 2).

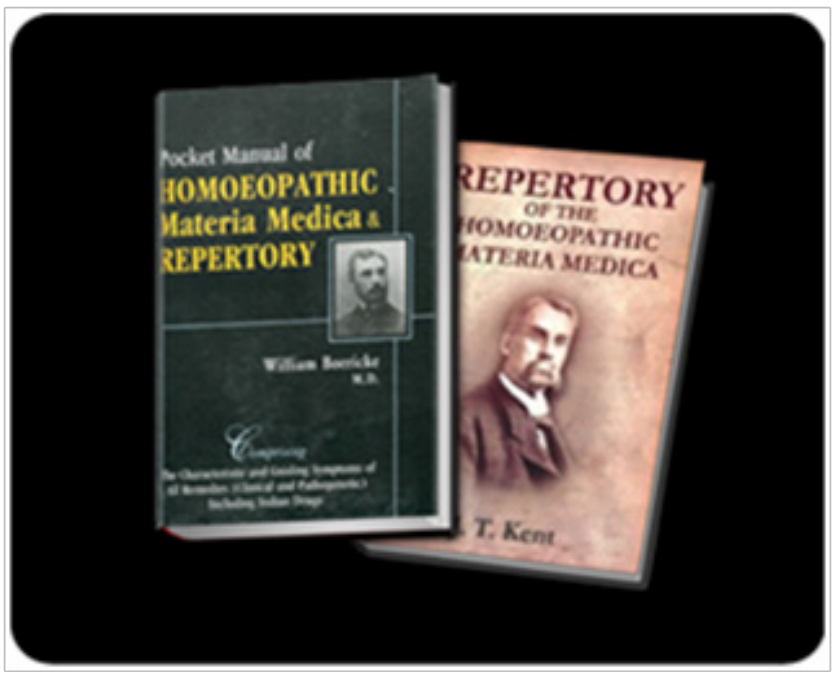

Figure 2 Materia Medica.

Provings or tests are the profile of specific symptoms produced by a substance in a healthy individual. These symptoms can be 
local such as the symptoms of Cinchona on joints, presenting concomitant (occurring at the same time) symptoms like Cinchona's fevers and shivers, and psychological/emotional as discussed below. The experimentation and the specific symptoms they manifest are reproducible and the same every time.

The provings are reproducible and the effects have been consistent over time. This type of data analysis started with Homeopathy and later became the basis of experimentation and scientific medical research.

Treating patients with the small amounts of these substances was next and Hahnemann established that by diluting these substances until there was minimal concentration of them in his treatments. He started treating symptoms of diseases based the toxicological effects earlier documented in the Provings and matching them to the diseased person's symptoms.

Based on these observations he formed the "Principle of Similars": a substance that produces symptoms of sickness in a healthy individual will have a curative effect when given in very small quantities to a diseased person exhibiting those symptoms. This concept is also known as "Like Cures Like".

Homeopathy is believed to stimulate the body's own healing processes by stimulating different systems including the immune system (Figure 3).

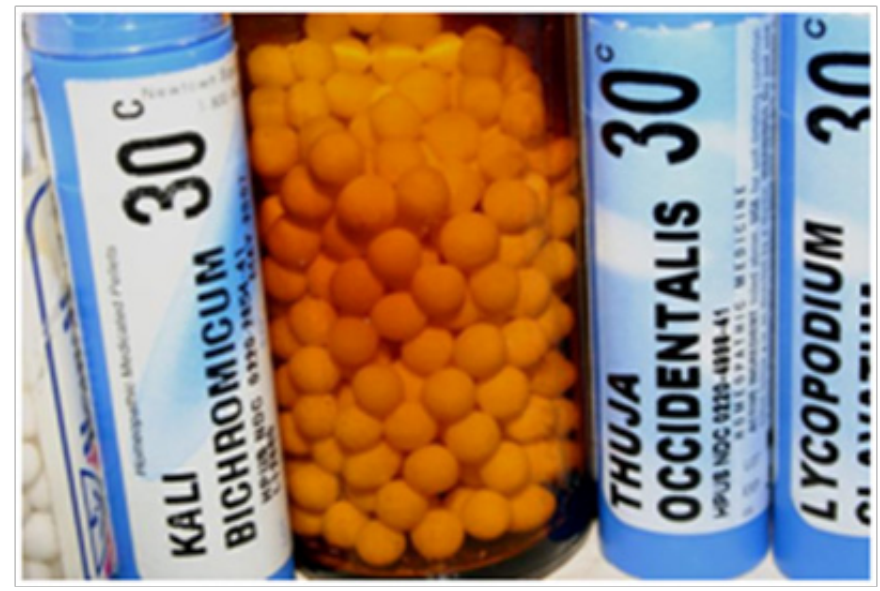

Figure 3 Homeopathic Medications.

\section{How does homeopathy work?}

The basic principle of Homeopathy, "Like Cures Like" has benefited conventional medicine over time. Vaccination for example is injecting small doses of a live attenuated virus or bacteria or their particles. The body then is encouraged to increase its immunity by using the small amount of these substances. Allergy shots are another example of this basic principle. For example, minimal doses of cat dander are injected to a person with severe allergies to cats to encourage their body reverse the severe allergic reaction, anaphylaxis, that can be initiated by being exposed to cats.

Anaphylaxis is a severe allergic reaction causing an inflammatory process, which produces swelling of the mucous membranes. In severe untreated cases this can cause wheezing and choking.

\section{How is homeopathy different than conventional medicine?}

Homeopathic medications are specific to the individual. A headache may be treated differently according to its location, sensation, modalities (aggravating and alleviating) and concomitant symptoms in the same individual at different times. So, a headache which radiates from the neck to the top of the scalp and to the eye and has a shooting pain will have a different homeopathic medication than one which starts in the sinus and radiates to the eye and has a congestive, throbbing sensation. Comparing this to conventional medicine a single medication may be used for both types of headaches, like Tylenol.

If not chosen properly the medication is not going to have an effect and this requires a rigorous training for the physician prescribing the homeopathic medication.

The specificity of Homeopathic medications renders them less or non-effective if not chosen properly. This is one of the reasons why it has been hard to design a medical study, which will prove their effectiveness. However, recent studies with proper selection of the medication have proven the effects of Homeopathic medication. A recent meta-analysis of three double blind clinical trials of diarrhea in 242 children ages 6 months to 5 years showed the duration of diarrhea of 3.3 days in the homeopathy group compared with 4.1 in the placebo group $(\mathrm{P}=0.008)$. Meta-analysis shows a consistent effect-size difference of approximately 0.66 day $(\mathrm{P}=0.008) .{ }^{1}$

\section{Medication or herb? What do we call homeopathy?}

I get asked this question a lot. Also, I hear patients referring to any alternative modality as Homeopathy.

Homeopathy uses substances found in nature. Herbs, animal parts, plants, and minerals are used to make Homeopathic medications. Highly diluted substances are carried in pill, syrup, drops, and cream forms. Thus, alternative medical treatments like acupuncture, supplements, herbs, are not Homeopathy.

Since these medications are highly diluted, they are free of side effects and usually a good substitute to the treatment of children and pregnant women.

\section{Homeopathy in action!}

Think of the effects a bee sting leaves on an individual. The area that was stung will get edematous, warm, red, and tender. Homeopathic preparation of Apis Mellifica will reverse the symptoms resembling a bee sting, like certain infections, acne, and skin issues.

\section{Apis mellifica}

In this instance the animal parts of a bee (Figure 4), which include Phospholipase A2 and Melittin (cause Histamine release a hormone for allergic reaction) and other peptides which cause mast cell degranulation (causes allergic reaction), are used as the raw material, called the Mother Tincture. The Homeopathic medication is made by diluting the mother tincture serially until very minimal amounts of the substance stays in the medicine. Due to the presence of those constituents in the mother tincture Apis Mellifica is also a treatment of choice for allergic reactions, runny nose, skin reactions, itching and edema (Figure 5). 


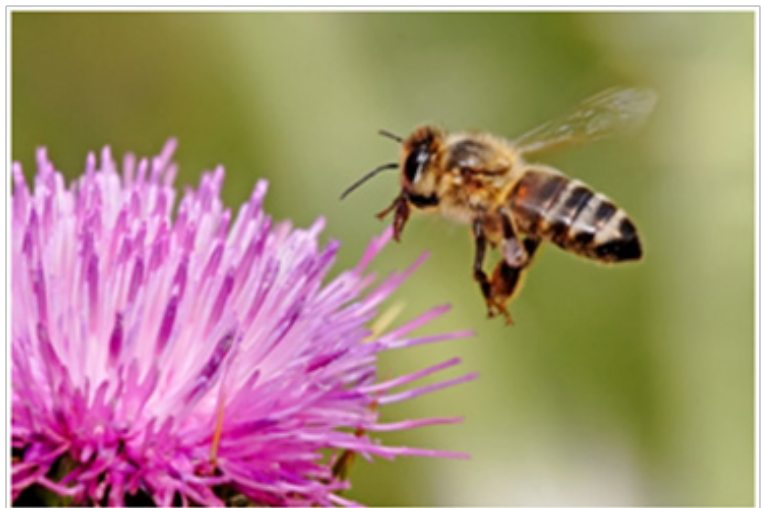

Figure 4 Apis Mellifica.

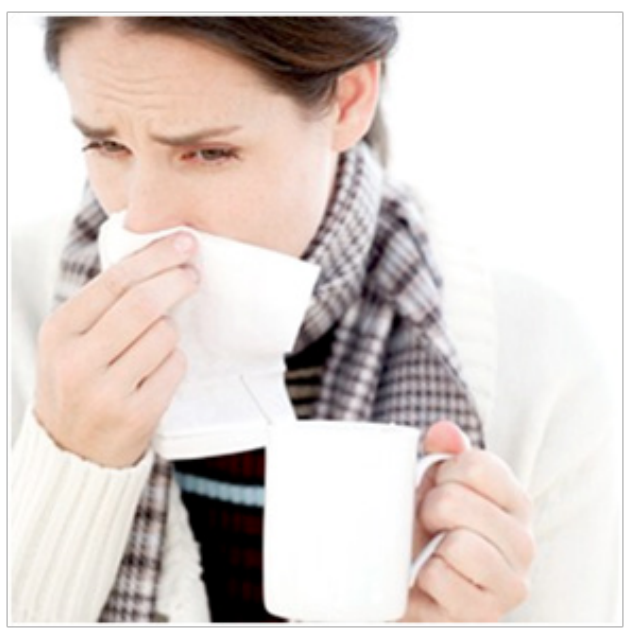

Figure 5 Allergies.

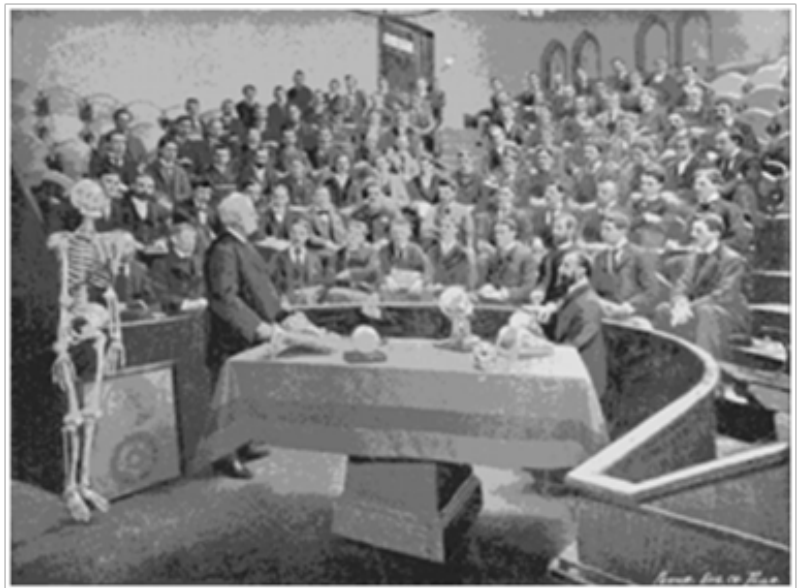

Figure 6 The anatomical amphitheater at Hahnemann Medical School, Philadelphia. The lecture is accompanied by demonstration of museum specimens.

\section{What does homeopathy treat?}

Homeopathy can be used to treat most illnesses, chronic or acute, like headaches, sinus and ear infections, heartburn, constipation, food poisoning, and different painful conditions. It can also be used adjunctively with conventional medicine without interacting or causing side effects.
More importantly, Homeopathy can be used for certain conditions that conventional medicine struggles with. Certain conditions like shooting pains, vertigo, tinnitus, to name a few, are treated by Homeopathic medications and comparing to the side effects of conventional medicine Homeopathic medications show superior effect in these cases.

\section{What doesn't homeopathy treat?}

All healing modalities, including conventional medicine, osteopathy, acupuncture, chiropractic, massage therapy, reiki, psychological and emotional therapies, and homeopathy have limitations.

Homeopathy was very popular in the United States due to its proven action. At a certain point there were 22 Homeopathic schools and 15,000 Homeopathic physicians in the United States. The reason Homeopathy lost its popularity was because it was used by nonmedical individuals who promised more than Homeopathy could offer to their patients and to the medical world.

Homeopathy is taught in medical school in Europe, and thus prescribed by physicians who know the limits of their treatment modalities and also know the superiority and effectiveness of conventional medicine in certain cases. This is what I always teach in my Homeopathic seminars with CEDH (center for education and development of clinical homeopathy) and workshops: "As physicians we have the responsibility of prescribing the best treatment for our patients keeping in mind the most important principle of medicine: first, does no harm. This means evaluating the patient medically first and forming a decision on what course of action to take. It is harmful to treat a patient presenting with the symptoms that might suggest an acute heart condition, for example, with only alternative medicine and not utilize our sophisticated emergency and critical care facilities."

During my years of practice I have not seen anyone treated for cancer by Homeopathy, although, I have used Homeopathic medication to reverse some of the side effects of chemotherapy. Neither have I had the experience with or heard any Homeopathic physician treating ADD/ADHD, and autism with proven success with Homeopathy, although, I have treated hyperactive and agitated children with Homeopathy with good success. In my experience other severe and chronic conditions like diabetes, schizophrenia, severe depression and bipolar disorder, chronic hypertension cannot be treated with Homeopathy.

Homeopathy Workshops articles will discuss the science of Homeopathy for the purpose of educating the public and healthcare professionals. During the course of these articles Homeopathy is explained with reference to history, experiments, scientific research, and my personal experiences with Homeopathy in my practice. Homeopathic medicines for certain conditions like insomnia, upper respiratory infections, PMS, menopausal symptoms, gastroenteritis, and other illnesses are discussed.

"The body is physical, structural, physiological, emotional, psychological, electro-chemical and spiritual. The future of medicine is about understanding this and getting back to looking inside the person rather than only at their disease." -Dr. Hakimi

\section{Acknowledgments}

None. 


\section{Conflict of interest}

The authors declare that there is no conflict of interest.

\section{Funding}

None.

\section{References}

1. Jacobs J, Jonas WB, Jiménez-Pérez M, et al. Homeopathy for childhood diarrhea: combined results and meta-analysis from three randomized, controlled clinical trials. Pediatr Infect Dis J. 2003;22(3):229-234. 